\title{
A Numerical Approach to solve Three-Parameter Matrix Eigenvalue Problems by Kronecker Product Method
}

\author{
Niranjan Bora \\ Dibrugarh University Institute of Engineering and \\ Technology (DUIET), \\ Dibrugarh University
}

\author{
Arun Kumar Baruah \\ Department of Mathematics \\ Dibrugarh University \\ Dibrugarh: 786004
}

\begin{abstract}
In this work it is intended to discuss three-parameter matrix eigenvalue problems and its numerical aspects. The problem is reduced into its corresponding one-parameter problems in tensor product space. Then applying kronecker product method eigenvalue and eigenvectors have been estimated. A numerical example is also presented in this paper.
\end{abstract}

\section{Subject Classification: 47A70, 65F15 (2010)}

\section{Keywords}

Multiparameter eigenvalue problems, tensor product space, kronecker product.

\section{INTRODUCTION}

The Multiparameter Eigenvalue problems (MEPs) is the generalization of classical one-parameter eigenvalue problems

$\mathrm{Ax}=\lambda \mathrm{x}$, where the problem is to find a $\mathrm{k}$-tuple values $\lambda=\left(\lambda_{1}, \lambda_{2}, \lambda_{3}, \lambda_{4} \ldots \ldots \ldots \lambda_{k}\right) \in C^{k}$ and non-zero vector $x_{i} \in C^{n_{i}}$ for $i=1,2, \ldots \ldots k$ such that

$$
\left(A_{i}-\sum_{j=1}^{k} \lambda_{j} B_{i j}\right) x_{i}, i=1,2, \ldots \ldots k
$$

where $\lambda_{i} \in R^{i} ; i=1,2,3, \ldots \ldots \ldots k$. are spectral parameters and $A_{i}, B_{i j} ; j=1,2,3 \ldots \ldots \ldots \ldots k$ are certain linear operators that act in Hilbert Spaces. The k-tuple $\lambda \epsilon C^{k}$ is called an eigenvalue and the tensor product $x=x_{1} \otimes x_{2} \otimes$ $x_{3} \ldots \ldots \ldots \otimes x_{k}$ is the corresponding (right) eigenvector. Similarly left eigenvector can also be defined.

MEPs involving several parameters arise in mathematical physics when the method of separation of variables is used to solve boundary value problems [1], more specifically those which lead to Mathieu functions. The eigenvalue problems for the matrices and differential equations have gained the general importance under more abstract settings. Numerical solution of MEP for matrices arises in the discretization of Multiparameter Sturm-Liouville eigenvalue problems in ordinary differential equations [2]. Matrix eigenvalue problems [1,3] involving several parameters comes from a large number of areas, such as chemistry, mechanics, dynamical systems, Markov chains, magneto-hydrodynamics, oceanography and economics.

The MEP have received attention not only by the Mathematicians in the recent years but also from the noted early investigators namely Bocher, Klein, Hibert, Roach etc,. The original motivation of MEP comes from the classical problem of solving boundary value problems for partial differential equations by the method of separation of variables
[4]. A vibrating membrane problem [Roach [5]] and a dynamical problem of a homogeneous beam loaded by a vertical load [Collatz [6]] are the typical examples in this regard. Indeed, MEPs arising from this source were investigated by Hilbert in the first decade of the last century. The first definitive account of Multiparameter Spectral Theory is due to Atkinson which he described in his books [1, 7]. This theory was first announced in 1972 and has undergone considerable development by Browne [8] and Kallstrom and Sleeman [9] both in the abstract case and in application to ordinary differential operators.

There are several numerical methods for the MEPs. Bohte [10] used Newton's method to find an eigenvalue pair. Müller [11] used the continuation method to compute eigenvalue curves starting from a given eigenvalue. Blum and Chang [12] derived the minimum residual quotient iteration (MRQI) for the problem $A x=\lambda B x+\mu C x$ (where $\lambda$ and $\mu$ are parameters) subject to $\|\mathrm{x}\|=1$ and $f(x)=0$, where $f$ is a real functional. $\mathrm{Ji}$, Jiang, and Lee [13] generalized their approach to the right definite two-parameter problem and derived the generalized Rayleigh quotient iteration (GRQI).Binding and Browne [14] described some variational methods for the solution of MEPs. Browne and Sleeman [15] used gradient method. Blum et al. $[16,17,18]$ considered the Multiparameter eigenvalue problem of one equation and presented a gradient method. Baruah [19] used three different methods for the numerical treatment of a two-parameter eigenvalue problem. Baruah and Konwar [20] showed five different techniques to obtain the starting values of the eigenpairs. Classification of MEP in elliptic, parabolic and hyperbolic problems by examples of matrix and integral equations are found in Collatz [6].

It has been observed that one-parameter problems have grained much more development both theoretically and numerically in comparison to MEPs. Moreover, numerical study on Multiparameter problems, particularly, twoparameter problems are also well investigated by many authors in terms of differential equations. But only a few authors has studied two-parameter matrix eigenvalue problem. This paper will concern about the numerical treatment of three-parameter eigenvalue problems in matrix equation by Knoneker Product Method, in which three-parameter eigenvalue problems will be reduced to corresponding oneparameter problems in tensor product space [21] and then applying numerical method on the resulting problem the eigenvalues and the corresponding eigenvectors will be estimated. 
The rest of the paper is organized as follows: Section 2 contains a brief description of three-parameter problem and the reduction of multiparameter problems to a system of oneparameter problems. In Section 3 a numerical example is presented. Finally, in Section 4 importance of the Knonecker Product Method is presented.

\section{REDUCTION OF THREE}

\section{PARAMETER EIGENVALUE PROBLEM} INTO A SYSTEM OF ONE-PARAMETER EIGENVALUE PROBLEMS

The problem considered is a three-parameter eigenvalue problem for matrix equations. Let $H_{1}=R^{p}, H_{2}=R^{q}$ and $H_{3}=R^{r}$ be finite dimensional Hilbert spaces, and $F=H_{l} \otimes$ $H_{2} \otimes H_{3}$ the Hilbert tensor product space. In each space $H_{j}, j=1,2,3$ existence of symmetric matrix operators assumed as $A_{1}, B_{11}, B_{12}, B_{13}: R^{p} \rightarrow R^{p} ; A_{2}, B_{21}, B_{22}, B_{23}$ : $R^{q} \rightarrow R^{q}$ and $A_{3}, B_{31}, B_{32}, B_{33}: R^{r} \rightarrow R^{r}$. The three-parameter problem considered is

$O_{1} u_{1}=A_{1}-\left(\lambda_{I} B_{11}+\lambda_{2} B_{12}+\lambda_{3} B_{13}\right) u_{1}=0$

$O_{2} u_{2}=A_{2}-\left(\lambda_{1} B_{21}+\lambda_{2} B_{22}+\lambda_{3} B_{23}\right) u_{2}=0$

$O_{3} u_{3}=A_{3}-\left(\lambda_{1} B_{31}+\lambda_{2} B_{32}+\lambda_{3} B_{33}\right) u_{3}=0$

An eigenvalue is defined to be a 3-tuple $\left(\lambda_{1}, \lambda_{2}, \lambda_{3}\right)$ of complex numbers for which there exists a non-zero decomposable element $u=u_{1} \otimes u_{2} \otimes u_{3}$. The necessary definiteness condition is given by

$\left[\begin{array}{lll}\left(B_{11} u_{1}, u_{1}\right) & \left(B_{12} u_{1}, u_{1}\right) & \left(B_{13} u_{1}, u_{1}\right) \\ \left(B_{21} u_{2}, u_{2}\right) & \left(B_{22} u_{2}, u_{2}\right) & \left(B_{23} u_{2}, u_{2}\right) \\ \left(B_{31} u_{3}, u_{3}\right) & \left(B_{32} u_{3}, u_{3}\right) & \left(B_{33} u_{3}, u_{3}\right)\end{array}\right] \geq 0$

for all $u_{1} \in H_{1}, u_{2} \in H_{2}, u_{3} \in H_{3}, u_{1} u_{2} u_{3} \neq 0$.

Problem (1) can be reduced to a system of three oneparameter problems in the space $F[1]$

$\Delta_{1} u=\lambda_{1} \Delta_{0} u, \quad \Delta_{2} u=\lambda_{2} \Delta_{0} u, \quad \Delta_{3} u=\lambda_{3} \Delta_{0} u$

$\Delta_{0}^{-1} \Delta_{1} u=\lambda_{1} u, \quad \Delta_{0}^{-1} \Delta_{2} u=\lambda_{2} u, \quad \Delta_{0}^{-1} \Delta_{3} u=\lambda_{3} u$

where

$\Delta_{0}=\left[\begin{array}{lll}\breve{B}_{11} & \breve{B}_{12} & \breve{B}_{13} \\ \breve{B}_{21} & \breve{B}_{22} & \breve{B}_{23} \\ \breve{B}_{31} & \breve{B}_{32} & \breve{B}_{33}\end{array}\right], \quad \Delta_{0}=\left[\begin{array}{ccc}-\check{A}_{1} & \breve{B}_{12} & \breve{B}_{13} \\ -\check{A}_{2} & \breve{B}_{22} & \breve{B}_{23} \\ -\check{A}_{3} & \breve{B}_{32} & \breve{B}_{33}\end{array}\right]$

$\Delta_{2}=\left[\begin{array}{lll}\check{A}_{1} & -\breve{B}_{11} & -\breve{B}_{13} \\ \check{A}_{2} & -\breve{B}_{21} & -\breve{B}_{23} \\ \check{A}_{3} & -\breve{B}_{31} & -\breve{B}_{33}\end{array}\right]$ and $\Delta_{3}=\left[\begin{array}{lll}-\breve{A}_{1} & \breve{B}_{11} & \breve{B}_{12} \\ -\check{A}_{2} & \breve{B}_{21} & \breve{B}_{22} \\ -\check{A}_{3} & \breve{B}_{31} & \breve{B}_{32}\end{array}\right]$

$u=u_{1} \otimes u_{2} \otimes u_{3}$

Here the operators denoted by $\tilde{A}_{l}, \breve{B}_{11} \breve{B}_{12} \quad \breve{B}_{13}$ in $F$ are the operators induced by $A_{1}, B_{11}, B_{12}, B_{13}$ in $H_{1}$. Similarly $\tilde{A}_{2}, \breve{B}_{21}$ $\breve{B}_{22} \quad \breve{B}_{23}$ and $\tilde{A}_{1}, \breve{B}_{31}, \breve{B}_{32}, \breve{B}_{33}$ are operators in $F$ induced by operators $A_{2}, B_{21}, B_{22}, B_{23}$ in $H_{2}$ and $A_{3}, B_{31}, B_{32}, B_{33}$ in $H_{3}$ respectively. Thus the induced operators can be written as

$\tilde{A}_{1}=A_{1} \otimes I_{2} \otimes I_{3}, \quad \tilde{A}_{2}=I_{1} \otimes A_{2} \otimes I_{3}, \quad \tilde{A}_{3}=I_{1} \otimes I_{2} \otimes A_{3}$.

$\breve{B}_{1 i}=B_{1 i} \otimes I_{2} \otimes I_{3}, \quad \breve{B}_{2 i}=I_{l} \otimes B_{2 i} \otimes I_{3}, \quad \breve{B}_{3 i}=I_{l} \otimes I_{2} \otimes B_{3 i}$ $i=1,2,3$.

$I_{i}$ are the identity operators in $H_{i}, i=1,2,3$.
The following results about the system (2) are due to [19]:

(a) The operators $\Delta_{0}, \Delta_{1}, \Delta_{2}, \Delta_{3}$ are symmetric.

(b) The operator $\Delta_{0}$ is positive definite.

(c) The operators $G_{i}=\Delta_{0}^{-1} \Delta_{i}, i=1,2,3$ commute.

The three eigenvalue problems are then given by

$\tilde{O}_{1}\left(\lambda_{1}, \lambda_{2}, \lambda_{3}\right) u=0, \tilde{O}_{2}\left(\lambda_{1}, \lambda_{2}, \lambda_{3}\right) u=0, \tilde{O}_{3}\left(\lambda_{1}, \lambda_{2}, \lambda_{3}\right) u=0$

Where $\tilde{O}_{i}, i=1,2,3$ denotes the induced operators of the operators $O_{i}$ defined in (2) and $u=u_{1} \otimes u_{2} \otimes u_{3}$. Concerning the connection between the system (2), (4), and (5), the following results are found in [1].

\subsection{Theorem}

Let $\left(\lambda_{1}, \lambda_{2}, \lambda_{3}\right)$ be an eigenvalue and $\left(u_{1}, u_{2}, u_{3}\right)$ a corresponding eigenvector of the system (2). Then $\left(\lambda_{1}, \lambda_{2}, \lambda_{3}\right)$ is an eigenvalue of the system (4), while $u=u_{1} \otimes u_{2} \otimes u_{3}$ is the corresponding decomposable eigenvector.

\subsection{Theorem}

Let $\left(\lambda_{1}, \lambda_{2}, \lambda_{3}\right)$ be an eigenvalue and ' $u$ ' a common eigenvector of the system (4). Then $\left(\lambda_{1}, \lambda_{2}, \lambda_{3}\right)$ is an eigenvalue and $u$ a common eigenvector of the system (5).

\subsection{Theorem}

Let $\left(\lambda_{1}, \lambda_{2}, \lambda_{3}\right)$ be an eigenvalue and ' $u$ ' an eigenvector of the system (5). Then $\left(\lambda_{1}, \lambda_{2}, \lambda_{3}\right)$ is an eigenvalue of the system (2). For eigenvectors the following relation is true:

Ker $\mathrm{O}_{1} \otimes \mathrm{Ker} \mathrm{O}_{1} \otimes \mathrm{Ker}_{3}=\mathrm{KerO}_{1} \otimes \mathrm{KerO}_{2} \otimes \mathrm{KerO}_{3}$

\subsection{Corollary}

Let $\left(\lambda_{1}, \lambda_{2}, \lambda_{3}\right)$ be a simple eigenvalue, then the corresponding eigenvector is decomposable.

From Theorems (2.1), (2.2) and (2.3) it follows that the eigenvalues and eigenvectors of the system (2) can be determined from the eigenvalues and eigenvectors of the system (4). Therefore, the solution of the three-parameter eigenvalue problems is reduced to the solution of three simultaneous one-parameter problems with common eigenvectors. The solution of one-parameter eigenvalue problems can be obtained by using known algorithms. In the light of the theorem by Slivnik at. all. [20], the following theorem can be proved.

\subsection{Theorem}

The eigenvectors of the problem $\Delta_{1} \mathrm{u}=\lambda_{1} \Delta_{0} \mathrm{u}$ can be chosen so that they are eigenvectors of the problem

$\Delta_{2} \mathrm{u}=\lambda_{2} \Delta_{0} \mathrm{u}$ or $\Delta_{3} \mathrm{u}=\lambda_{3} \Delta_{0} \mathrm{u}$ and vice versa.

Proof: As $A$, is positive definite it can be decomposed in the form (Cholesky decomposition)

$$
\Delta_{0}=L L^{T}
$$

where $L$ is a lower triangular operator. By denoting $L^{T} u=y$ the system (2) can be written in the form

$L^{-1} \Delta_{1}\left(L^{-1}\right)^{T} y=\lambda_{1} y, L^{-1} \Delta_{2}\left(L^{-1}\right)^{T} y=\lambda_{2} y$ and $L^{-1} \Delta_{3}\left(L^{-1}\right)^{T} y=$ $\lambda_{3} y$,

$$
\text { Or } P_{1} y=\lambda_{1} \mathrm{y}, P_{2} y=\lambda_{2} \mathrm{y} \text { and } P_{3} y=\lambda_{3} \mathrm{y}
$$

where $P_{i}=L^{-1} \Delta_{\mathrm{i}} L^{T}, i=1,2,3$. 
Evidently $P_{1}, \quad P_{2}$ and $P_{3}$ are symmetric. From the commutativity of the operators $\mathrm{G}_{1}, \mathrm{G}_{2}$ and $\mathrm{G}_{3}$ it follows that $P_{1}$,

$P_{2}$ and $P_{3}$ are also commutative. Since commuting symmetric operators have a common system of eigenvectors, and hence the theorem.

\section{NUMERICAL EXAMPLE}

The numerical example considered is a three-parameter problem defined by equations (7) as below. The problem is so constructed that it satisfies the definiteness condition (3).

$$
\begin{aligned}
\left(\begin{array}{lll}
1 & 0 & 0 \\
0 & 1 & 0 \\
0 & 0 & 1
\end{array}\right) \mathrm{u}_{1}= & \left\{\lambda_{1}\left(\begin{array}{ccc}
10 & 0 & 1 \\
0 & 15 & 0 \\
1 & 0 & 14
\end{array}\right)+\lambda_{2}\left(\begin{array}{ccc}
-1 & 0 & 0 \\
0 & -1 & 0 \\
0 & 0 & -1
\end{array}\right)\right. \\
& \left.+\lambda_{3}\left(\begin{array}{ccc}
-2 & 0 & 0 \\
0 & 2 & 0 \\
0 & 0 & 2
\end{array}\right)\right\} \mathrm{u}_{1}
\end{aligned}
$$

$$
\begin{aligned}
\left(\begin{array}{lll}
6 & 0 & 0 \\
0 & 7 & 0 \\
0 & 0 & 8
\end{array}\right) \mathrm{u}_{2}=\left\{\lambda_{1}\left(\begin{array}{ccc}
-2 & 0 & 0 \\
0 & -3 & 0 \\
0 & 0 & -4
\end{array}\right)+\lambda_{2}\left(\begin{array}{ccc}
10 & 0 & 0 \\
0 & 20 & 0 \\
0 & 0 & 30
\end{array}\right)\right. \\
\left.+\lambda_{3}\left(\begin{array}{lll}
5 & 0 & 0 \\
0 & 2 & 0 \\
0 & 0 & -2
\end{array}\right)\right\} \mathrm{u}_{2} \\
\left(\begin{array}{ccc}
2 & 0 & 0 \\
0 & 3 & 0 \\
0 & 0 & 4
\end{array}\right) \mathrm{u}_{3}=\left\{\lambda_{1}\left(\begin{array}{lll}
5 & 0 & 0 \\
0 & 3 & 0 \\
0 & 0 & 4
\end{array}\right)+\lambda_{2}\left(\begin{array}{ccc}
12 & 0 & 0 \\
0 & 13 & 0 \\
0 & 0 & 11
\end{array}\right)+\right. \\
\left.\lambda_{3}\left(\begin{array}{ccc}
4 & 0 & 0 \\
0 & -4 & 0 \\
0 & 0 & -3
\end{array}\right)\right\} \mathrm{u}_{3}
\end{aligned}
$$

The operators $\Delta_{0}, \Delta_{1}, \Delta_{2}$ and $\Delta_{3}$ of the equations (4) in the reduced one parameter problems are given by

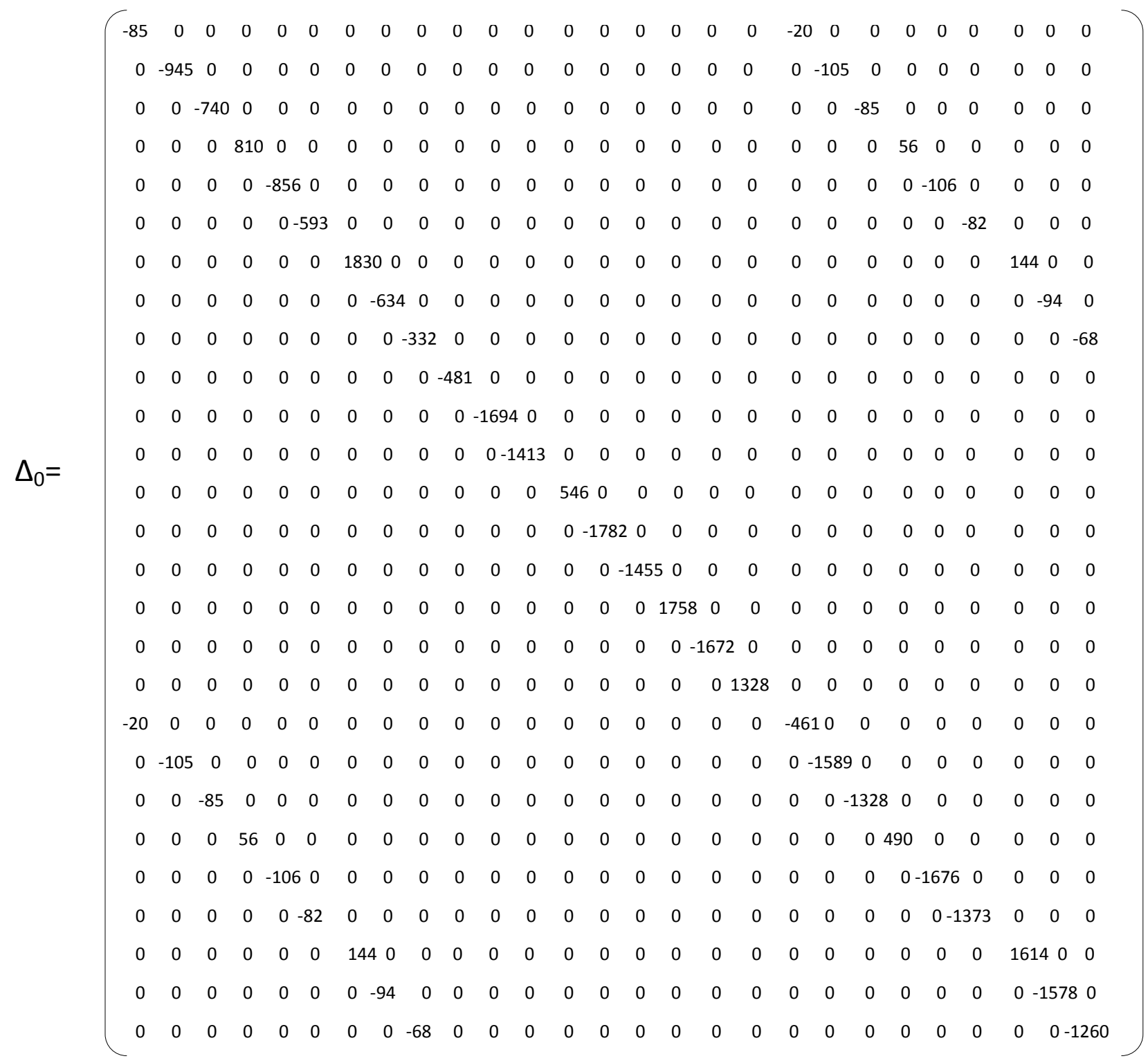

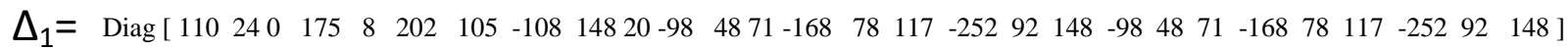




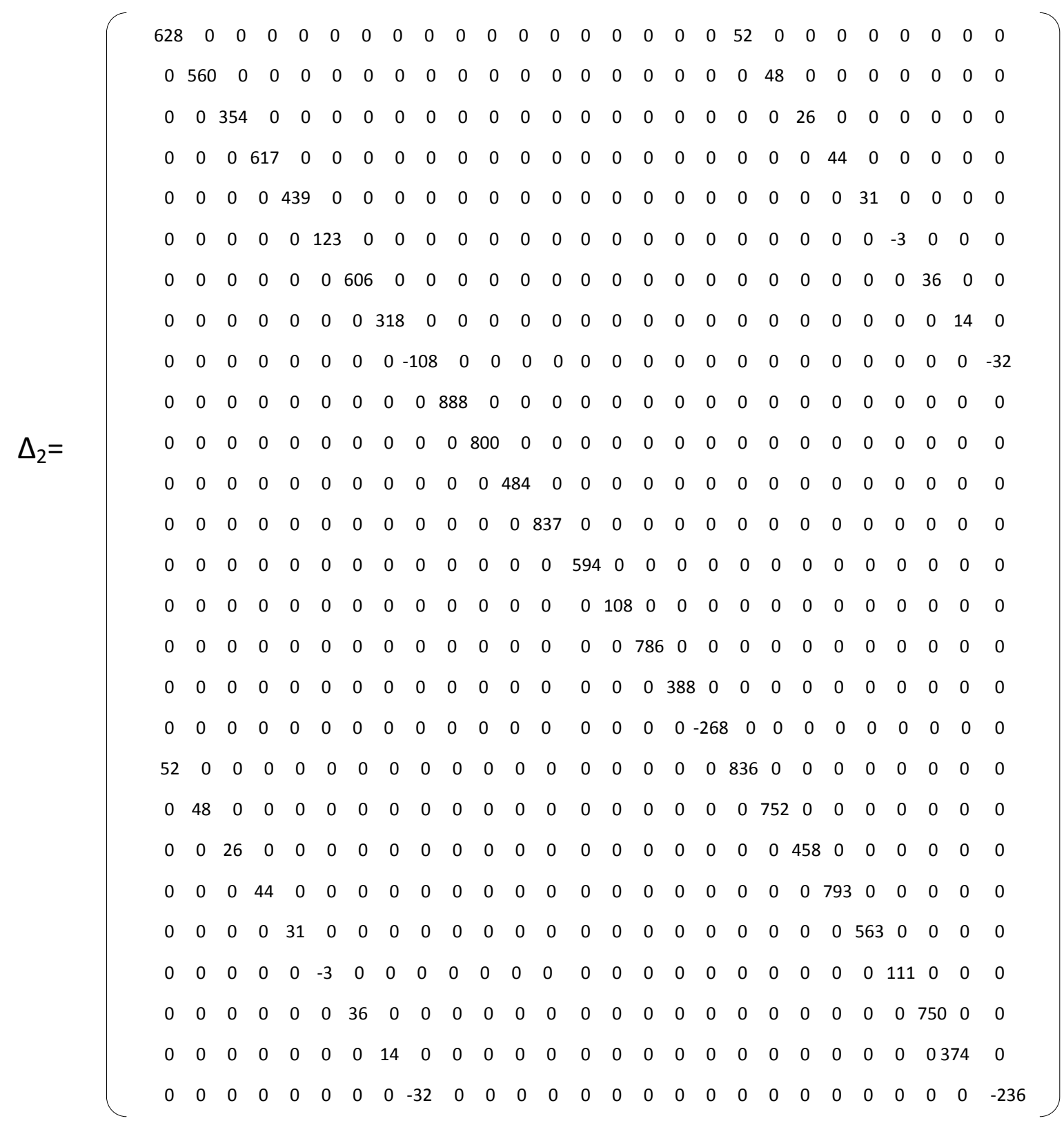




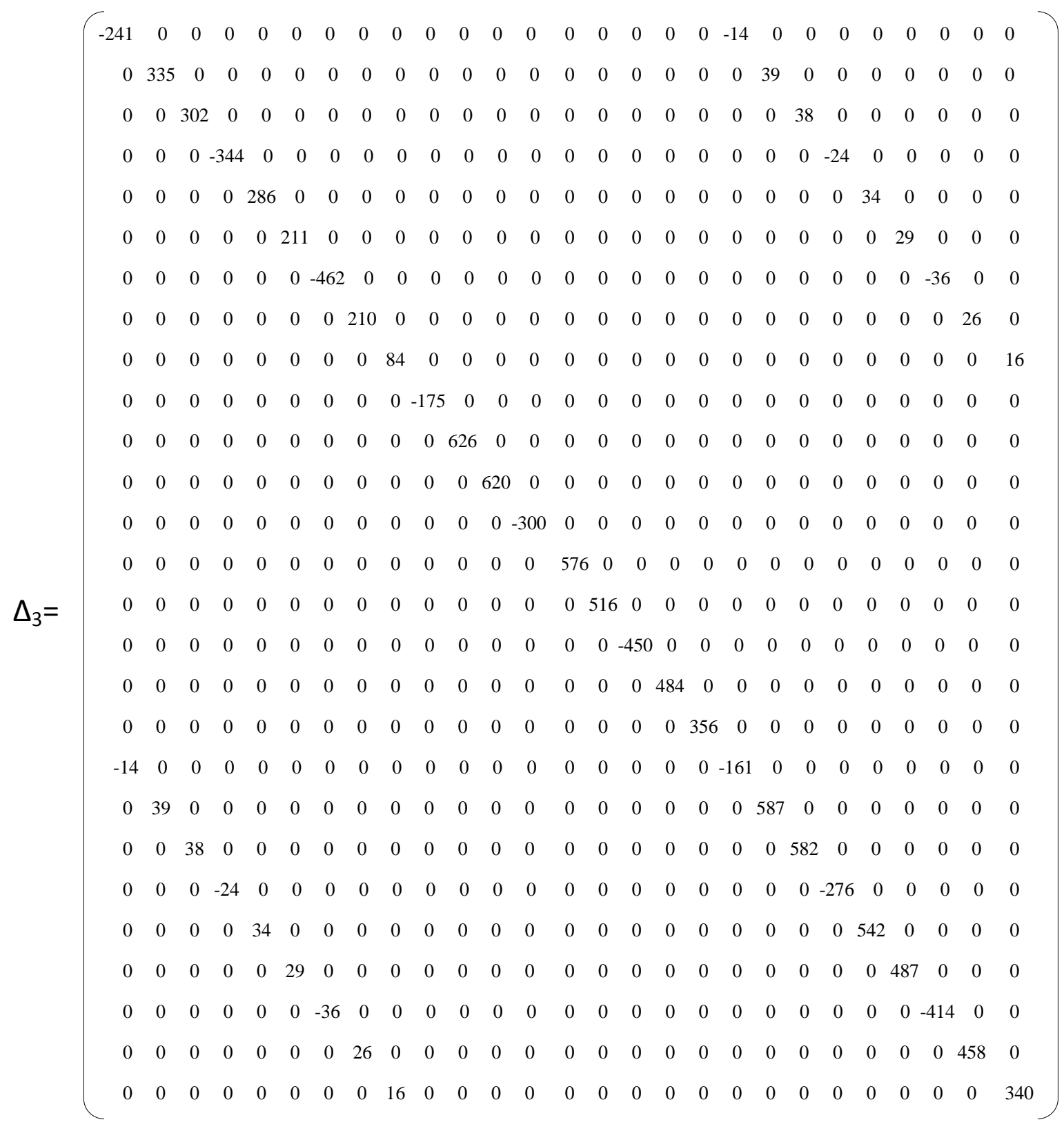

Table-1: Table for eigen 3-tuples and the corresponding eigenvectors

\begin{tabular}{|c|c|}
\hline Eigen 3-tuples & Eigenvectors \\
\hline$(-1.3056,2.8542,-7.4306)$ & 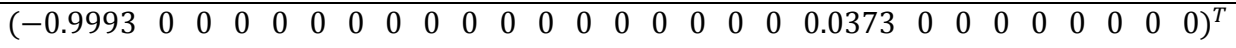 \\
\hline$(-0.2387,-0.4077,-0.480)$ & $\left(\begin{array}{llllllllllllllllllllllllllll}0 & 0 & -0.9966 & 0 & 0 & 0 & 0 & 0 & 0 & 0 & 0 & 0 & 0 & 0 & 0 & 0 & 0 & 0 & 0 & 0 & 0.0822 & 0 & 0 & 0 & 0 & 0 & 0\end{array}\right)^{T}$ \\
\hline$(-0.0533,-0.4383,-0.3448)$ & $\left(\begin{array}{llllllllllllllllllllllllllll}0 & 0 & 0.0334 & 0 & 0 & 0 & 0 & 0 & 0 & 0 & 0 & 0 & 0 & 0 & 0 & 0 & 0 & 0 & 0 & 0 & 0.9994 & 0 & 0 & 0 & 0 & 0 & 0\end{array}\right)^{T}$ \\
\hline$(-0.0804,-0.3546,-0.0742)$ & $\left(\begin{array}{llllllllllllllllllllllllllll}0 & 0 & 0 & 0 & 0 & 0 & 0 & 0 & 0 & 0 & 0 & 0 & 0 & 0 & 1.000 & 0 & 0 & 0 & 0 & 0 & 0 & 0 & 0 & 0 & 0 & 0 & 0\end{array}\right)^{T}$ \\
\hline$(-0.1114,-0.2681,0.2018)$ & $\left(\begin{array}{llllllllllllllllllllllllllll}0 & 0 & 0 & 0 & 0 & 0 & 0 & 0 & 0 & 0 & 0 & 0 & 0 & 0 & 0 & 0 & 0 & 1.000 & 0 & 0 & 0 & 0 & 0 & 0 & 0 & 0 & 0\end{array}\right)^{T}$ \\
\hline
\end{tabular}


MATLAB software is used to calculate the eigenvalue and the corresponding eigenvectors of the three one-parameter problem of the equation (4) separately. The number of common 3-tuples of this system will be $3^{3}=27$. Here five different eigen 3-tuples and their corresponding common eigenvectors are shown in Table - 1 above.

\section{CONCLUSION}

In this paper, the three-parameter matrix eigenvalue problem is reduced into its corresponding one-parameter problems in tensor product space. Then applying Knonecker Product Method eigenvalues and the corresponding eigenvectors have been estimated with the help of MATLAB software. Though in the present work the three-parametric case is considered, it can be extended to more than three-parameter problems but the computational efforts as well as time will be high for higher dimensional matrix equations. As this method is based on a sound theoretical concept, so the convergence to the exact eigenvalues and to the eigenvectors is almost guaranteed.

\section{ACKNOWLEDGMENTS}

The authors are indebted to A. Bharali, Department of Mathematics, Dibrugarh University for helpful discussions.

\section{REFERENCES}

[1] Atkinson, F.V., 1972. "Multiparameter Eigenvalue Problems", Vol. I, (Matrices and Compact Operators) Academic Press,New York.

[2] Hua Dai ${ }^{\mathrm{a}}$, 2007. "Numerical methods for solving multiparameter eigenvalue problems," International Journal of Computer Mathematics, 72:3, 331-347.

[3] Sleeman, B.D., 1978, "Multiparameter Spectral Theory in Hilbert Space," Pitman Press, London.

[4] Volkmer, H., 1988, "Multiparameter Problems and Expansion Theorems," Lecture Notes in Math.1356, Springer-Verlag, New York.

[5] Roach, G.F., "A classification and reduction of general Multiparameter problems," Technical report, GeorgAugust-Universität Göttingen

[6] Collatz, L., 1968. "Multiparameter eigenvalue problems in inner product spaces", J.Compu. and Syst. Scie., 2, 333-341.

[7] Atkinson, F.V., and Mingarelli, A. B., 2010. "Multiparameter Eigenvalue Problems: Sturm-Liouville Theory", CRC Press.
[8] Browne, P. J., 1972. "A multiparameter eigenvalue problem," Journal of mathematical Analysis and Applications 38, 553-568.

[9] Kallstrom, A., and Sleeman, B. D., 1977. "Multiparameter Spectral Theory," Ark. Mat.15, 93-99.

[10] Bohte. Z., 1982. "Numerical solution of some twoparameter eigenvalue problems", Slov. Acad. Sci. Art., $17-28$.

[11] Müller, R. E., 1982. "Numerical solution of multiparameter eigenvalue problems",Z. Angew. Math. Mech., 62, 681-686.

[12] Chang, A.F., Blum, E.K., 1978. "A numerical method for the solution of the double eigenvalue problem", J. Inst. Math.appl. 22, 29-42.

[13] Ji, X. R., Jiang, H., and Lee, B. H. K. "A Generalized Rayleigh Quotient Iteration for Coupled Eigenvalue Problems", manuscript.

[14] Binding, P. and Browne, P.J., 1981. "Spectral properties of two parameter problems".Proc. Roy. Soc. Of Edinburgh 89A, 157-173.

[15] Browne, P. J., and Sleeman, B.D., 1982. "A numerical technique for multiparameter eigenvalue problems," IMA J. of Num. Anal. 2, 451-457.

[16] Curtis, A. R., and Blum, E.K., 1978. "A convergent Gradient method for matrix eigenvector-eigentuple problems", Numer. Math., 31, 247-263.

[17] Geltner, P. B., and Blum, E. K., 1978. "Numerical solution of eigentuple-eigenvector problems in Hilbert spaces by a gradient method", Numer. Math., 31, 231 246.

[18] Rodrigue, G.H., and Blum, E.K., 1974. "Solution of eigenvalue problems in Hilbert spaces by a gradient method". J. Comput. System Sci. 2, 220-237.

[19] Baruah, A.K., 1987. "Estimation of eigenelements in a two-parameter eigenvalue problem," Ph.D thesis, Dibrugarh University.

[20] Baruah, A. K. and Konwar, J., 2001, "A Numerical Technique to a Two-Parameter Eigenvalue Problem", was published in the Proceedings of the $46^{\text {th }}$ International Congress of ISTAM held at Regional Engineering College, Hamirpur, H.P.during, 132-139.

[21] Slivnik, T. and Tomsit, G., 1984. "A numerical method for the solution of two-parameter eigenvalue problems," Institute of Mathematics, Physics, and Mechanics, E. K. University of Ljubljana, 61000 Ljubljana, Yugoslavia. 